\title{
Toward Flexibility in Sensor Placement for Motion Capture Systems: A Signal Processing Approach
}

\author{
Roya Haratian, Richard Twycross-Lewis, Tijana Timotijevic, and Chris Phillips, Member, IEEE
}

\begin{abstract}
Human body motion can be captured by body area sensor networks. Accurate sensor placement with respect to anatomical landmarks is one of the main factors determining the accuracy of motion-capture systems. Changes in position of the sensors cause increased variability in the motion data, so isolating the characteristic features that represent the most important motion patterns is our concern. As accurate sensor placement is time-consuming and hard to achieve, we propose a signal processing technique that can enable salient data to be isolated. By using functional principal component analysis (f-PCA), we compensate for the variation in data due to changes in the on-body positioning of sensors. More precisely, we investigate the use of f-PCA for filtering and interpreting motion data, whilst accounting for variability in the sensor origin. Data are collected through a marker-based motion capture system from two designed experiments based on human body and robot arm movement. Results show differences between similar actions across different sessions of marker wearing with random changes in position of sensors. After applying the f-PCA filter on the data, we show how uncertainties due to sensor position changes can be compensated for.
\end{abstract}

Index Terms - Functional principal component analysis, measurement variability, motion capture.

\section{INTRODUCTION}

$\mathbf{O}$ N-BODY sensors can be used for capturing human motion. The motion of a body can be thought of as a collection of time series streams describing the joint angles, which is called the motion data. Motion data can be used in applications such as animation, sports biomechanics, rehabilitation, and so on. In many applications, the human body is approximated by a collection of articulated limbs that form a kinematic tree. Determining the anthropometry of the individual subject is called model calibration. Accurate sensor placement with respect to anatomical landmarks and location determination of joint centers with regard to these sensors are two important aspects of model calibration in motion capture. Special care must be taken to achieve levels

Manuscript received August 13, 2013; accepted October 5, 2013. Date of publication October 23, 2013; date of current version January 7, 2014 The associate editor coordinating the review of this paper and approving it for publication was Prof. Ricardo Gutierrez-Osuna.

R. Haratian, T. Timotijevic, and C. Phillips are with the School of Electronic Engineering and Computer Science, Queen Mary University of London, London E1 4NS, U.K. (e-mail: roya.haratian@eecs.qmul.ac.uk; tijana.timotijevic@eecs.qmul.ac.uk; chris.phillips@eecs.qmul.ac.uk).

R. Twycross-Lewis is with the Centre for Sports and Exercise Medicine, William Harvey Research Institute, Queen Mary University of London, London E1 4DG, U.K. (e-mail: r.twycross-lewis@qmul.ac.uk).

Color versions of one or more of the figures in this paper are available online at http://ieeexplore.iee.org.

Digital Object Identifier 10.1109/JSEN.2013.2286994 of reliability sufficient to justify the results of motion capture systems despite measurement variability.

Variability in movement patterns plays a fundamental role in motion analysis and its influence on the analysis of motion data should be taken into account. Inconsistencies due to placement errors of on-body sensors can come from three primary sources: the technicians responsible for placing the sensors/markers, the measurement system, and the subject under evaluation [1]. Variability is defined by the sum of variances from each independent source [2]. Sensor placement among technicians is the largest source of variability [3]. Particular care should be taken to ensure that sweating, rapid movements and the placement of markers during different trials and sessions on the subject's body, do not affect sensor/marker positioning according to the marker placement guidelines.

Changes in the position of sensors can also be influenced by their fitting on/within smart sensing garments, which in turn affect performance. While appropriate for continuous use, sensing garments need to consider the wearers' comfort and fitting requirements [4], which often hamper measurement performance. Tight-fitting cloths are frequently perceived uncomfortable while most sensors and applications benefit from a close textile-body coupling. For less-tight garments, selecting appropriate sensor positions is essential and it is often performed empirically by expert opinion [5], which may not be optimal, or generalized to a population aggravating measurement variability [6]. This issue arises with intelligent textile technologies for applications in wearable computing and health monitoring, smart human-machine interface, and so forth [7]. Silicon flexible skins have been introduced to address flexibility in sensing garments [8]. While such techniques try to develop a new technology for intelligent sensing, in our research we explore techniques that can permit current commercial off-the-shelf technologies to be used more effectively. We elect to use signal processing for its flexibility with smart sensing.

The reliability of measurements is directly affected by the sensor placement during different sessions. If experimental errors conceal important motion deviations, meaningful information will be lost. On the other hand, if the limitations of the motion capture methods are not understood, small deviations may be considered meaningful, thereby leading to over interpolation [9]. Every time that a subject tries to carry out the same movement, a certain amount of variation may be registered between different sessions of marker wearing. Variability between sessions was found to be much higher 
than within-session variability because of the high probability of differences in the marker placement that arise [2].

Sensor misplacement and thus anatomical landmark mislocation have a substantial effect on joint kinematics and angles. Sensitivity of joint kinematics variables to anatomical landmark misplacement has been reviewed in [10]. In [11] it is shown substantial differences can arise in determining anatomical landmark location between the expert and selfmarking operators. The "greater trochanter" location was found by two groups of operators to be more than $20 \mathrm{~mm}$ apart and all pelvic anatomical landmarks were determined with more than $10 \mathrm{~mm}$ difference. In [12] it has been studied that hip joint centre mislocation of $30 \mathrm{~mm}$ in the anterior-posterior direction generates a mean error on the flexion/extension moment of about $22 \%$. Furthermore, it has been shown in [13] that anatomical landmark position uncertainties result in the observation of physiological knee motions such as the "screwhome" mechanism even when such motion did not occur. These uncertainties can lead to erroneous clinical interpretation of the estimation and thus misdiagnosis.

As discussed increased variability in motion data can be associated with arbitrary changes in position of sensors, so discovering the characteristic features that represent the main motion pattern is our concern. Standard data analysis techniques, which determine the mean and standard deviation of time series data, summarize motion data in single patterns that is the average behaviour and show deviations as possible errors by standard deviation bands. Such techniques severely reduce the information gleaned from the raw data, and may discard much important information [14]. In particular, the results does not account for the information that may be inherent in all the variations apparent in the data. When different sessions of marker wearing are averaged, information can be lost and the averaged curve does not closely resemble any of the individual curves.

To facilitate analysis, eliminate collinearity and present only the essential structures contained within the data, multivariate statistical analysis has proved to be a powerful tool [15]. However, the extent of data loss remains a matter of concern. Among multivariate statistical techniques, linear transformations are computationally easy to perform. With linear transformations, the use of functional techniques may provide additional insight into differences in motion patterns. Treating the data as functions preserves all the information contained in the raw data. Functional principal component analysis (f-PCA) is an effective tool for the study of human motion in modelling motion curves by identifying hidden combinations and relationships between variables [16].

The basic philosophy of functional data analysis on human motion data is the belief that the best unit of information is the entire observed function rather than a string of numbers. It is assumed that human motion data are supposed to have an underlying functional relationship governing them [17]. A central idea in functional data analysis is smoothness which implies that adjacent values in time are linked and it is unlikely that these values will differ largely [18]. Since f-PCA, which is an extension of traditional PCA, provides a means of identifying and examining the main source of variability of a set of curves, it is useful for analysing human movement data where variability plays a key role [19].

Principal Component Analysis (PCA) is a multivariate statistical technique that provides an orthogonal projection of the data onto a lower dimensional linear space, known as the principal subspace. It can be used as a decorrelation technique by computing a new, much smaller set of uncorrelated variables, i.e. principal components (PCs) [20]-[21]. Each new variable is a linear combination of the original ones. All the principal components are orthogonal to each other, so there is no redundant information. All remaining principal components are defined similarly, so that the lowest order components normally account for very little variance and can usually be ignored [22]-[23]. In other words, PCA can be defined as a linear projection that minimizes the average projection cost, defined as the mean squared distance between the data points and their projections [24]. PCA has already been used as an effective tool in motion analysis for extracting variation features in motion data [25]-[26].

In f-PCA, there is an eigenfunction associated with each eigenvalue, while in PCA there is eigenvector. These eigenfuctions describe major variational components. It also permits extracting loadings and scores. Loadings are the correlation coefficients between variables and components. Scores are the contributions of the principal components to each individual variable [14]. Motion data can be decorrelated by projection on the eigenfuctions. After projection, stochastic components can be separated from deterministic components, by subtracting either one or the other from the signal.

In our previous work [27], a PCA based filtering technique has been used to compensate for the effect of changes in the position of sensors. Conversely, in this paper we focus on f-PCA, and by designing and performing an experiment with a robot arm we assess the validity and the applicability of the technique that we introduce. We study and compensate for the uncertainties in the data due to sensor position changes. To reflect the true nature of motion data variability, we investigate the use of f-PCA for filtering and interpreting motion data, while accounting for their positional variability. In Section 2, after summarising the problem, our method is introduced by describing motion data acquisition process, designed experiments, data analysis and a brief review of functional principal component analysis. There are two sets of experiments, one on a human subject and the other on a robot arm to validate the human subject experiment in a more controlled movement. Results and a discussion are then presented in Section 3. Our paper is concluded in Section 4.

\section{METHOD}

We use functional principal component analysis to discover the characteristic features that represent the main patterns of motion, while reducing the variability in motion data due to arbitrary changes in position of sensors. To describe the compensation procedure, we first consider the process of motion data acquisition and then the data processing techniques used in our study. Our subsequent experiments comprise two scenarios: motion capture of a human subject 
and a robot arm. The second scenario, which utilizes a more controlled movement in comparison to the first scenario, validates the experiment on the human subject. Our data processing techniques are then discussed.

\section{A. Motion Data Acquisition Process}

We use a commercial optical motion capture system named Codamotion. The system is a general-purpose 3D Motion tracking system. The measurement unit contains three prealigned solid-state cameras which track the position of a number of active markers, i.e. infra-red LEDs, in real-time. The LED markers are powered from small battery boxes, drive boxes, which are available in two sizes, 2-marker or 8-marker. Sampling rates are selectable from $1 \mathrm{~Hz}$ up to $200 \mathrm{~Hz}$, dependent on the numbers of markers in use. Each Codamotion scanner unit contains three special cameras which detect infra-red pulses of light emitted by the Codamotion markers and locate the marker positions with very high resolution and linearity. The cameras are rigidly mounted in the scanner units so that the system can be pre-calibrated. The calibrated system measures the positions of markers within a three dimensional coordinate system that is fixed in relation to the scanner unit. The active range of the capturing system is $1.5-5.2 \mathrm{~m}$ from the scanners and follows a Gaussian distribution function so that optimal visibility occurs at a distance of approximately $3 \mathrm{~m}$ from each scanner. All the experiments are carried out in this range in accordance with the motion capture system setup in the laboratory. The process of system alignment is done by defining the $\mathrm{X}, \mathrm{Y}$, and $\mathrm{Z}$ axes of the reference coordinate system before performing each experiment [28]. The maximum measurement time depends on a combination of the sampling rate and the total number of markers being tracked. As the number of markers decreases, the maximum measurement time can be increased proportionally for a given sampling rate. The angular resolution of each camera is about 0.002 degrees; this results in a lateral position resolution of about $0.05 \mathrm{~mm}$ at 3 metres distance (horizontally and vertically), and a distance resolution of about $0.3 \mathrm{~mm}$ [28].

For human motion capture experiments, the long axis of the measurement unit defines the direction of the $\mathrm{X}$ axis, normally a horizontal line parallel to the walkway. The perpendicular line from the unit defines the positive $Y$ axi. The other perpendicular (vertical) line defines the $\mathrm{Z}$ axis (positive up) as is shown in Fig. 1. The marker numbers specified in Fig. 2 are the recommended sets; different numbers may be used as long as the appropriate names are assigned to the markers in the motion analysis setup. The geometrical properties of each body segment are derived, generally speaking, from three noncollinear points bearing particular anatomical relationships to the given segment.

There are several standard marker-sets for placing markers on the human body such as Cleveland Clinic, Saflo, Helen Hayes, Codamotion, and so forth. The Cleveland Clinic marker set uses a rigid triad of markers in a plane parallel to the long axis of the bone to capture the motion of the thigh and shank in 3 dimensions. The Saflo marker-set consists of a total body marker-set with 19 retro-reflective markers fixed

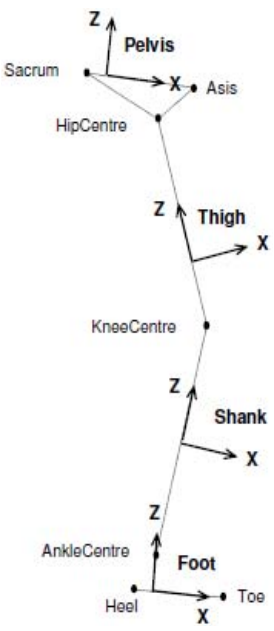

Fig. 1. Segment embedded coordinate frames according to the gait set up of Codamotion marker-set [28].

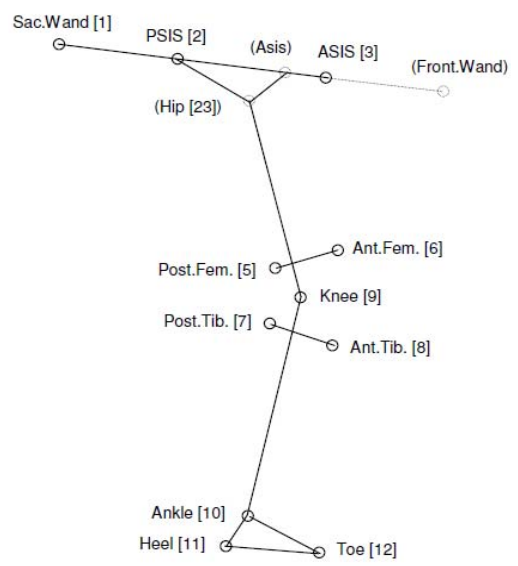

Fig. 2. Position of markers on human body according to the gait set up of Codamotion marker-set [28].

on specific anatomic landmarks. The Helen Hayes marker set is a relatively simple set of external markers developed for time-efficient video analysis of lower extremity kinematics. The original configuration of 13 markers minimizes the patient preparation and data acquisition time and reduces the number of trajectories that must be tracked or edited. All methods listed are used for clinical gait analysis. For bilateral gait, the recommended Codamotion marker-set comprises a total of 22 standard markers as shown in Fig. 2 for the right side of the body. Markers shown in parentheses () are optional [28]. The marker sets determine ankle and knee joint centres and segment coordinate systems by means of a marker on a post or wand protruding from the anterior aspect of the thigh and shank, and by single markers placed over the lateral aspect of the joint flexion/extension axis.

For the purpose of the current investigation in our experiments with controlled motion capture a robotic arm [29] was used to measure the effect of random marker placement errors during controlled rotations of one rigid body segment with respect to another. The robot has a positional repeatability of $\pm 0.02 \mathrm{~mm}$, stemming from its high precision when performing repetitive actions. When defining rigid body segments for three dimensional kinematic analysis, a minimum of three 
markers are required per segment, two markers define the longitudinal axis of the segment (in our laboratory, we define the longitudinal axis as the $\mathrm{Z}$ axis; anterior-posterior axis is the $\mathrm{X}$ axis and the medio-lateral axis is the $\mathrm{Y}$ axis) and a third marker is placed off centre from the $\mathrm{Z}$ axis to define the segment as a rigid body so that all three markers are noncolinear [30].

This arrangement of markers on rigid segments serves two functions. First: it enables us to define an Embedded Vector Basis (EVB) for the segment whereby each axis is mutually orthogonal (a process known as Gram-Schmidt orthogonalisation) [31], therefore allowing us to measure rotations using Euler angles. Second: the embedded axes of the local segment co-ordinate system are anatomically meaningful [32]. Euler angles are expressed through three sets of 3 by 3 matrices, with each matrix describing the rotation of a specific axis. In the case of our robotic arm segments, the rotation matrices, $\mathbf{R}$, used to describe rotation in each axis were:

$$
\begin{aligned}
& \mathbf{R}_{\mathbf{x}}=\left[\begin{array}{ccc}
1 & 0 & 0 \\
0 & \cos \theta & -\sin \theta \\
0 & \sin \theta & \cos \theta
\end{array}\right] \\
& \mathbf{R}_{\mathbf{y}}=\left[\begin{array}{ccc}
\cos \phi & 0 & \sin \phi \\
0 & 1 & 0 \\
-\sin \phi & 0 & \cos \phi
\end{array}\right] \\
& \mathbf{R}_{\mathbf{z}}=\left[\begin{array}{ccc}
\cos \psi & -\sin \psi & 0 \\
\sin \psi & \cos \psi & 0 \\
0 & 0 & 1
\end{array}\right]
\end{aligned}
$$

Non-cummulative matrix multiplication in the order Z,Y,X results in the decomposition matrix,

$$
\mathbf{R}_{\mathrm{zyx}}=\mathbf{R}_{\mathrm{x}} \mathbf{R}_{\mathrm{y}} \mathbf{R}_{\mathrm{z}}
$$

which correspond to the direction cosines between axes of two rigid body segments [32]. Equation (4) is called a decomposition matrix because the angles of rotation are decomposed into their projections onto the axes of the laboratory global co-ordinate system.

\section{B. Designed Experiments}

A full analysis of an individuals' motor behaviour should involve the evaluation of an appropriate number of individual repetitions. We used ten human subjects in ten sessions of marker wearing. There were inadvertent changes in the position of markers for each session while following the standard marker set. The process of measurements in each session involves instrumenting the legs and pelvis with active markers according to the Codamotion marker-set to perform the motion capture. Ten healthy human subjects, five male (weight $69 \pm 7.9 \mathrm{~kg}$, height $170.2 \pm 3.4 \mathrm{~cm}$, age $26.6 \pm 1.5$ years, BMI $23.8 \pm 1.9$ ) and five female (weight $56.4 \pm 6.9 \mathrm{~kg}$, height $167.2 \pm 3.2 \mathrm{~cm}$, age $25.6 \pm 1.5$ years, BMI $20.2 \pm 2.4$ ) were recruited for the instrumented gait analysis. Feigned "inadvertent changes" in position of markers in radius of $2 \mathrm{~cm}$ were made for each of ten sessions of marker wearing. We used a random number generator to generate random positions for sensors within a radius of $2 \mathrm{~cm}$ around body landmarks. Each session consisted of six trials. In each trial the subject

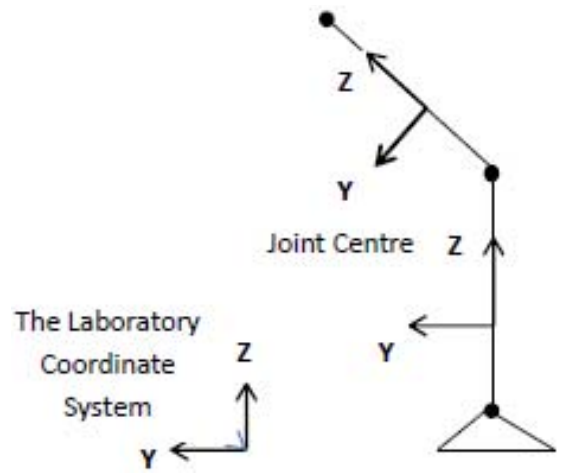

Fig. 3. Segment embedded coordinate frames for the robot arm.

walks from the start point to an end point while the motion capture system captures the subject's motion. Each trial lasts for five seconds and the sampling frequency of the system is $200 \mathrm{~Hz}$. The subject was asked to walk at normal walking speed. This walking speed is maintained as far as possible whilst different marker placements are made across different sessions of marker wearing.

For motion analysis of the robot arm which was divided into two rigid body; the upper segment defined as rigid body 1 (RB1); the lower defined as rigid body 2 (RB2). On RB1, markers were placed at either end of the segment, $3 \mathrm{~cm}$ from the end and defined the longitudinal $\mathrm{Z}$ axis of RB1. A marker was placed over the hinge joint between RB1 and RB2. This marker served as the upper marker for the $\mathrm{Z}$ axis marker for RB2 as well as the third, off-centre, marker required to define $\mathrm{RB} 1$ of the local coordinate system within the Codamotion software. As with RB1, RB2 was defined by two markers along the length of the segment and two further markers were used to define the RB2 segment local coordinate system (Fig. 3). The same configuration of markers was used on both sides of each segment and the local coordinate axis system for each segment was defined using a set of orthogonal axes.

The arm was programmed to rotate for 130 degree with markers in the described reference position as shown in Fig. 4. Measurements were recorded over intervals of fifteen seconds. The motion of the robot arm was recorded while the markers were in correct position to provide reference motion data. Then each marker was moved in a random direction at distances of 1 or $2 \mathrm{~cm}$ from the initial reference positions on the same plane of either segment. Twenty sets of data were collected: ten sets with markers randomly positioned $1 \mathrm{~cm}$ from the initial reference positions and ten sets of data with markers set randomly at a distance of $2 \mathrm{~cm}$ from the initial reference positions. In order to control rotation data as much as possible, movement was restricted to one degree of freedom; that is, the only movement was rotation of $\mathrm{RB} 1$ with respect to RB2 in one fixed plane. This movement was analogous to flexion-extension in the human arm. All other movements were constrained in order to reduce any measured confounding rotation data from orthogonal planes from the axis of rotation that we measured. 


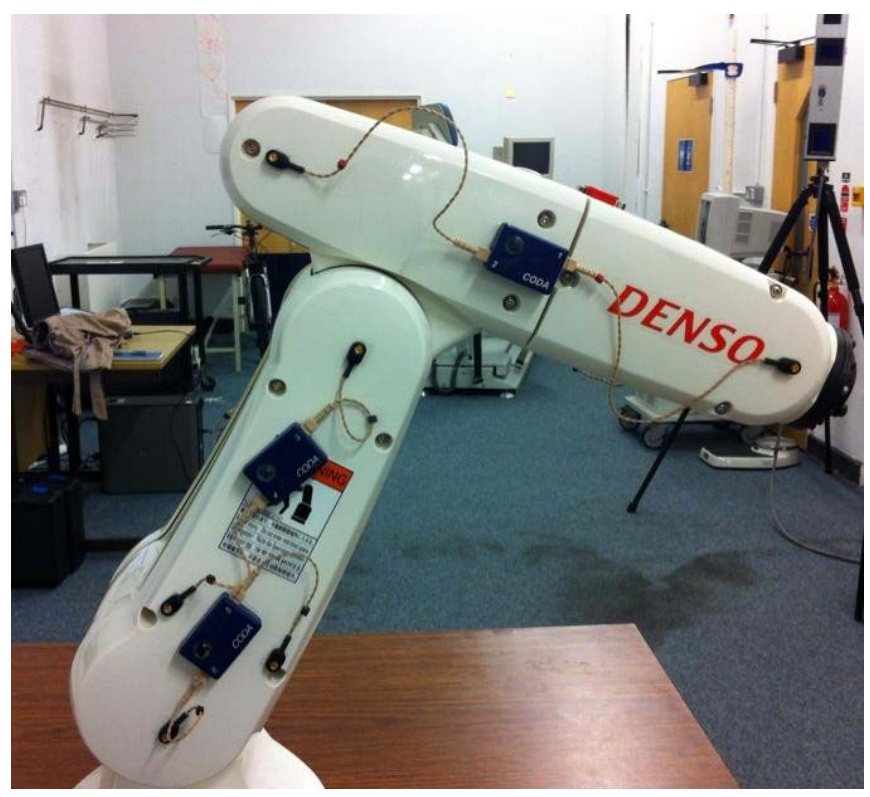

Fig. 4. Placement of markers which are attached to drive boxes positioned toward or on the mid-section of each rigid body in such a way that the robot arm could be modelled as two rigid body segments.

\section{Data Analysis}

Walking sequences were segmented into cycles. Each cycle comprises two steps. We asked a subject to walk in a certain time interval and divide the action into cycles. Each cycle is identified as the interval from initial contact of one step to initial contact of the following step of the ipsilateral limb, with a demarcation marker placed at toe off to define both stance and swing phases of the gait cycle. The same protocol was also used to mark the gait cycle for the contralateral limb so that the gait cycle was marked for both limbs. We use consecutive right heel contacts to separate each stride.

For the robot arm experiment, all joints with the exception of the flexion/extension joint of the robot arm were constrained; therefore, our experiment considered movement with one degree of freedom. We collected data from cyclic movement of the robot arm whereby each cycle is identified as the interval from maximal flexion to the following maximal flexion of the unconstrained flexion/extension joint. By using the $\mathrm{Z}$ axis position changes of Marker \#1 which is the proximal marker to the grabber, data cycles can be determined. Segmenting motion data into cycles almost always results in data cycles of differing lengths due to differences in motion speed.

In action recognition, identifying features during action sequences with different speeds or different numbers of samples in each cycle is an important issue. In such cases time normalization is necessary before or during the recognition process. Each cycle should be normalized so it is represented by the same number of samples. Linear and nonlinear time normalization using dynamic time warping are the most common technique that can be used for this purpose. Linear time normalization linearly converts the trajectory's time axis from the experimentally-recorded time units to an axis representing of the gait cycle [33].

\section{Functional Principal Component Analysis}

To find the dominant modes of variation in the data, and tease apart the deterministic and stochastic components of movement patterns, f-PCA can be a useful tool usually after subtracting the mean from each observation. It allows for separation of the main and residual components of a data set. Viewing consistent features as coherent components imply the mechanisms generating these common structures follow deterministic rules otherwise they would not be consistent/coherent. In contrast the residual components often contain a degree of randomness or stochasticity.

Functional principal component analysis is an extension of the traditional multivariate PCA statistical technique, where the principal components are represented by functions rather than vectors. The upper limit number of principal components in the multivariate case is the number of variables, while in f-PCA the number of eigenfunctions is equal to minimum of $K$, which is the number of basis functions, and $N$ which is the number of variables [18]. The number of basis functions should be less than or equal to the number of sampled data points, $n$. An exact representation is achieved when $K=n$. The smaller $K$ is and the better the basis functions reflect certain characteristics of the data, the less computation that is required. However, if we make $K$ too small, we may miss some important aspects of the function that we are trying to estimate. A larger $K$ provides a better fit to the data.

In the first step, we should fit function to the data. To fit a function to our data, we use a set of functional building blocks $\emptyset_{k}, k=1,2, \ldots, K$, called basis functions which are combined linearly (5). That is, a function $x(t)$ defined in this way is expressed as follows, and called a basis function expansion.

$$
x(t)=\sum_{k=1}^{K} c_{k} \emptyset_{k}
$$

Parameters $c_{k}$, are the coefficients of the expansion. The matrix expression of $N$ functions will be like $\mathbf{X}(t)=\mathbf{C} \emptyset(t)$, where $\mathbf{X}(t)$ is a vector of length $N$ containing the function $x_{\mathrm{i}}(t)$, and the coefficient matrix $\mathbf{C}$ has $N$ rows and $K$ columns. To compute these coefficients with more careful consideration of measurement error, there are two strategies. The two methods used for computing the coefficients more accurately are smoothing by regression analysis and by roughness penalties. In the first approach, it can be achieved by defining data fitting as the minimization of the Sum of Squared Errors, SSE, and considering the error model as follows,

$$
y_{j}=x\left(t_{j}\right)+\varepsilon_{j}=\mathrm{C}^{\prime} \emptyset(\mathrm{t})+\varepsilon_{j}=\emptyset^{\prime}\left(t_{j}\right) \mathrm{C}+\varepsilon_{j}
$$

Using matrix notation let the $n$-vector $\mathbf{y}$ contain the $n$ values to be fitted, vector $\varepsilon$ contains the corresponding true residual values, and $n$ by $k$ matrix $\emptyset$ contains the basis function values $\emptyset_{k}\left(t_{j}\right)$. Then we have $\mathbf{y}=\emptyset \mathbf{c}+\varepsilon$. The least-square estimate of the coefficient vectorc is

$$
\hat{c}=\left(\emptyset^{\prime} \emptyset\right)^{-1} \emptyset^{\prime} \mathbf{y}
$$

The roughness penalty approach uses a large number of basis functions but at the same time imposing smoothness by 
penalizing some measure of function complexity. A measure of roughness of the fitted curves, can be the square of the second derivate of a function $x$ at argument value $t$ which is called curvature at $t$.Thus, a measure of a function's roughness is the integrated squared second derivative or total curvature,

$$
\operatorname{PEN}_{2}(x)=\int\left[D^{2} x(t)\right]^{2} \mathrm{~d} t .
$$

Consequently the roughness penalized fitting criterion can be as (9), where $\lambda$ is the smoothing parameter specifying the emphasis on the second term penalizing curvature relative to goodness of fit quantified in the sum of squared residuals in the first term.

$$
F(\mathbf{c})=\sum_{j}\left[y_{j}-x\left(t_{j}\right)\right]^{2}+\lambda \operatorname{PEN}_{2}\left(\sum_{k=1}^{K} c_{k} \emptyset_{k}\right)
$$

By defining the $K$ symmetric roughness penalty matrix as $\mathbf{R}=\int \emptyset(\mathrm{t}) \emptyset^{\prime}(t) \mathrm{d} t$, We can express the estimate of the coefficient vector $\mathbf{c}$ as follows,

$$
\hat{c}=\left(\emptyset^{\prime} \emptyset+\lambda \mathbf{R}\right)^{-1} \emptyset^{\prime} \mathbf{y}
$$

After calculating the coefficients and deriving the fitted functions into our data, we calculate the sample variancecovariance function, $v(s t)$, which is defined as follows,

$$
v(s, t)=N^{-1} \sum_{i}^{N} y_{i}(s) y_{i}(t)
$$

The functional eigenequation is

$$
\int v(s, t) \xi(t) d t=\rho \xi(s),
$$

where $\rho$ is eigenvalue and $\xi(s)$ is an eigenfunction of the variance-covariance function. The eigenfunction which is called principal component weight function, $\xi_{1}(\mathrm{~s})$, can be found by (13).

$$
\begin{aligned}
& \text { Maximize } \sum_{i} f_{i 1}^{2} \\
& \text { Subject to } \int \xi_{1}^{2}(s) d s=\left\|\xi_{1}\right\|^{2}=1,
\end{aligned}
$$

where the principal component score, is defined as

$$
f_{i 1}=\int \xi_{1}(s) x_{i}(s) d s .
$$

A non-increasing sequence of eigenvalues $\rho_{1} \geq \rho_{2} \geq \ldots \geq \rho_{k}$ can be constructed stepwise by requiring each new eigenfunction computed in step 1 , to be orthogonal to those computed in the previous steps,

$$
\begin{aligned}
\int \xi_{j}(t) \xi_{l}(t) d t & =0, \quad j=1, \ldots, l-1 \\
\int \xi_{l}^{2}(t) d t & =1 .
\end{aligned}
$$

Solving the eigenequation for extracting functional Principal Components essentially reduces to performing Singular Value Decomposition (SVD) of (16). The columns of $\Delta$ contain the eigenfunctions (f-PCs) evaluated at time $t$ and $\Lambda$ contains the corresponding eigenvalues. The notation $\Delta^{T}(t)$ is used to identify that we are dealing with functions.

$$
v(s, t)=\Delta(s) \Lambda \Delta^{T}(t)
$$

To tease apart the deterministic and stochastic components of movement patterns, f-PCA can be used as a filtering technique, especially when partitioning signals into deterministic and stochastic components. This is achieved by subtracting either one or other from the signal and can be regarded as filtering the noise or the common parts, respectively.

As the effect of random changes in position of sensors causes a random effect on the motion data, to tease apart this effect from main and coherent component of movement, we partition the data into two elements, $\vec{x}_{i}{ }^{\text {(global) }}$ and $\vec{x}_{i}{ }^{\text {(filtered) }}$ as shown in (17);

$$
\begin{aligned}
\vec{x}_{i} & =\vec{x}_{i}^{(\text {global })}+\vec{x}_{i}^{(\text {filtered })} \\
& =\sum_{n=1}^{L<N} \xi_{n}(\mathrm{t}) f_{i}^{(n)}+\sum_{n=L+1}^{N} \xi_{n}(\mathrm{t}) f_{i}^{(n)}
\end{aligned}
$$

where we assume the number of basis functions are more than the number of variables. The sum of the dominant principal components weight functions is given by $\vec{x}_{i}$ (global), so the filter characteristic depends on the data. The number of modes that define the global pattern influences the filtered pattern.

After applying functional PCA on our data, we consider the main source of variation in data by keeping the most relevant principal component weight function and removing the rest from the f-PCA domain. After this stage, the data will be returned to the first domain whilst removing eigenfunctions with less variation. We apply this data-driven filter to the motion data of two sets of experiments on human subject and robot arm to separate out the effect of random changes in sensors position from main motion pattern by keeping the dominant modes of variation in the data whilst considering the proportion of corresponding eigenvalues to the total variance.

\section{RESUlTS AND DISCUSSION}

Kinematics variables were acquired for several motion capture sessions of the human body and the robot arm. Positions of sensors in 3D space are measured in accordance to the laboratory coordinate system. The captured sensors' positions in 3D space will differ depending on where the subject starts to walk. Since joint angles are descriptive of motion and do not vary with the change of the position of the subject with respect to the reference point, we choose to focus on angles of joints and the effect of sensors' position changes on derived angles. For experiments with the human body these variables are the angles of the pelvis, hip, knee, ankle, and foot in the X, Y, and $\mathrm{Z}$ axes. Kinematics variables of each marker wearing session were averaged over six trials of each session to eliminate the effect of factors that are irrelevant to differences in position of sensors in each session. The cause of these factors could be different walking speed, different ways of walking because of the tiredness of the subject and so on.

The aforementioned kinematics variables prior to f-PCA filtering are shown in Fig. 5. The differences which are shown between the variables of different sessions are due to inadvertent changes in position of sensors. The placement 

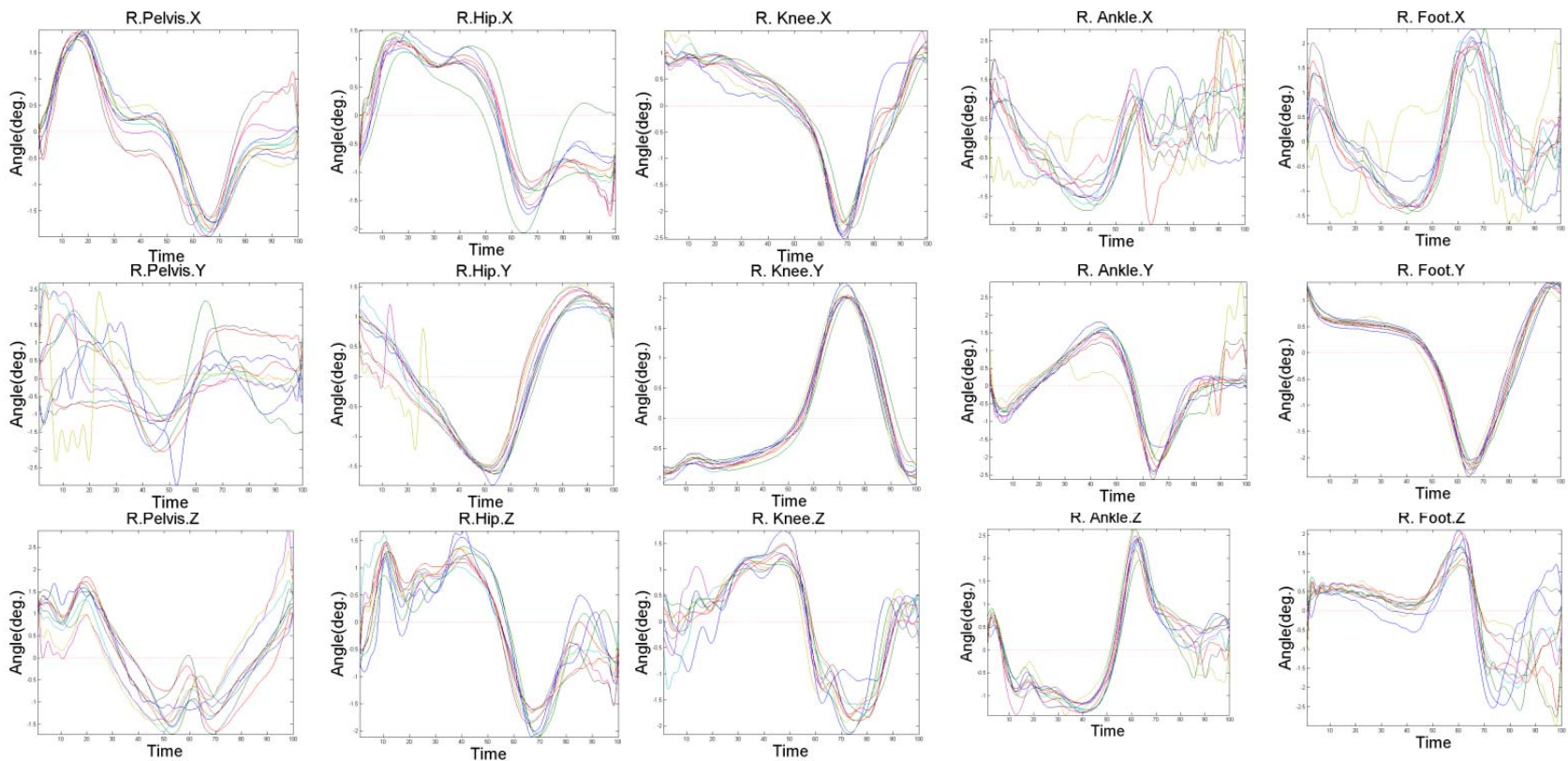

Fig. 5. Kinematics variables of 10 marker wearing sessions, respectively the normalized angles of pelvis, hip, knee, ankle, and foot in $\mathrm{X}$, Y, and $\mathrm{Z}$ planes separately from each other before applying the filtering technique.
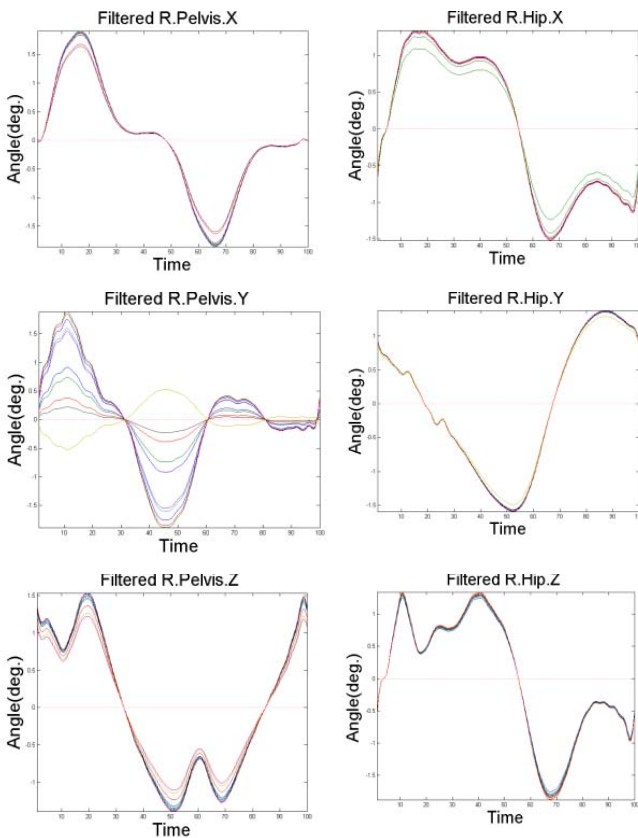
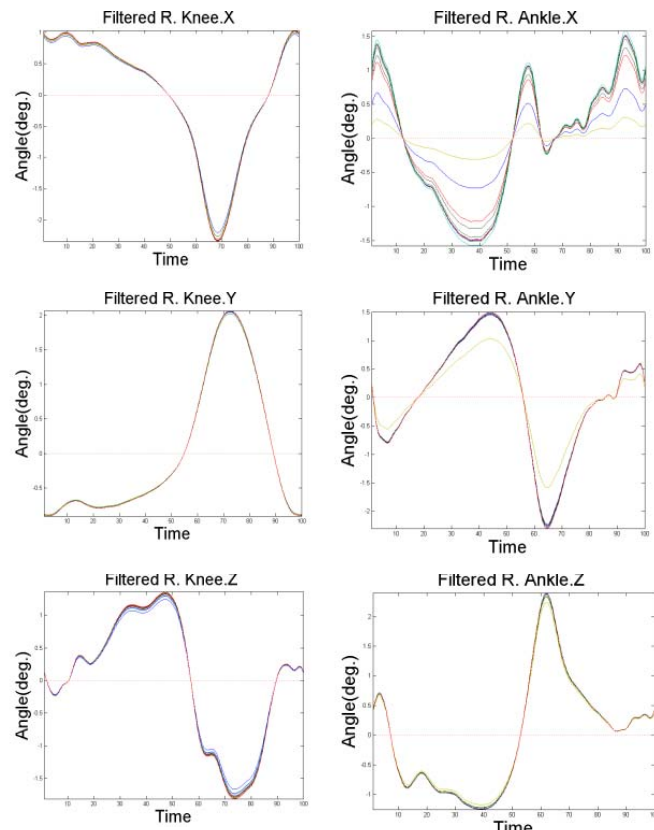

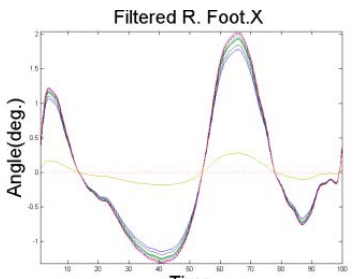

Filtered R. Foot.Y
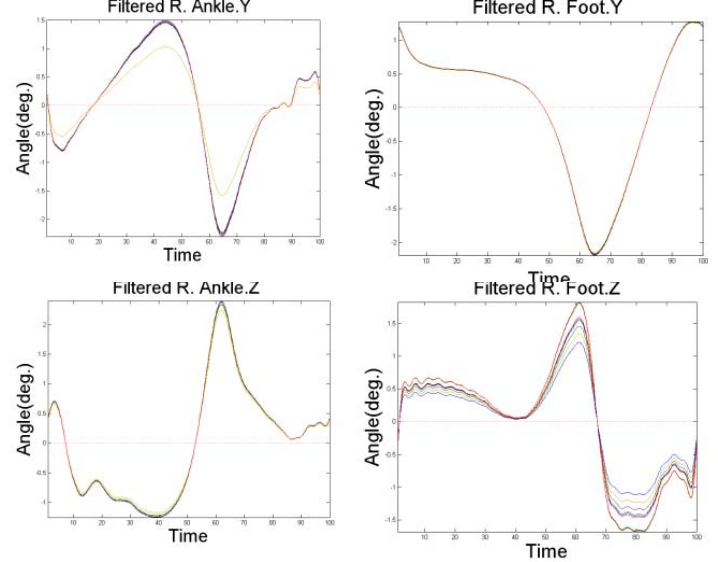

Fig. 6. Kinematics variables of 10 marker wearing sessions, respectively the normalized angles of pelvis, hip, knee, ankle, and foot in $\mathrm{X}$, $\mathrm{Y}$, and $\mathrm{Z}$ planes separately from each other after applying the filtering technique.

changes are random on all $\mathrm{X}, \mathrm{Y}$, and $\mathrm{Z}$ axes and they were made within a radius of $2 \mathrm{~cm}$ from the correct position which simulates the effect of technician marker placement errors across different sessions. Fitting Fourier basis functions into data from each session, we partition our initial cohort of 150 functions to a set of 10 groups, in which each group contains 15 function to describe each joint kinematic variables.

Each lot, therefore, contains one of the 15 kinematics variables in a cycle for each of the 10 sessions. By applying the proposed filtering technique on these lots, we obtain the functional principal component functions for each kinematic variable. The most dominant mode of variation is retained, and the remainder is eliminated, we thus preserve the principle variations in data and eliminate the effect of inadvertent changes in the position of the sensors. Finally, after deleting the non-dominant mode of variation, the functions are returned into initial domain by using the inverse f-PCA transform.

Figures 5 and 6 show motion averaged over 10 trials of 10 sessions on a human subject data pre and post f-PCA filtering. Using the technique on the motion data of ten subjects with ten of marker wearing sessions in each case, shows the mean variance between curves typically decreases by $95.76 \%$. The minimum averaged improvement is $88.15 \%$ for pelvic angle in the $\mathrm{Y}$ direction and the maximum $99.59 \%$ for knee angle in the 
TABLE I

Variation ARound the Reference Action While 1 AND $2 \mathrm{~cm}$ RANDOM CHANGES IN Position OF SENSORS BEFORE AND After ApPlying the TeChNiQUe

\begin{tabular}{c|c|c}
\hline & $\begin{array}{c}1 \mathrm{~cm} \text { Random } \\
\text { Changes }\end{array}$ & $\begin{array}{c}2 \text { cm Random } \\
\text { Changes }\end{array}$ \\
\hline $\begin{array}{c}\text { Variation before applying the } \\
\text { technique(deg.) }\end{array}$ & 2.02 & 5.69 \\
\hline $\begin{array}{c}\text { Variation after applying the } \\
\text { technique(deg.) }\end{array}$ & 0.76 & 2.10 \\
\hline
\end{tabular}

$\mathrm{Y}$ direction. It can be readily determined that f-PCA reduces variability associated with marker placement error. This was confirmed in the second experiment using robot arm.

The movement of the robot arm was analogous to flexionextension in the human arm. All other rotations were constrained to eliminate any movement in planes orthogonal to the measured plane. The movement was restricted to 1 degree of freedom in order to control rotation data as much as possible. After applying the technique we can see that the effect of random changes in position of sensors is reduced. We compared the motion data with data from reference marker positioning. As the radius of random changes in the position of sensors was increased, the variation in motion data from the reference data also increased as it is shown in Table 1.

Variation has been calculated as summation of differences between data of motion capture sessions from the reference motion capture data, divided by the number of samples. Because of high accuracy of the robot arm we are sure that the variation in data from the reference motion data is only due to random changes in position of sensors. Variability in marker placement error was reduced by $63 \%$ in both $1 \mathrm{~cm}$ and $2 \mathrm{~cm}$ random marker placement error experiments (Table 1). As the radius of random changes in position of sensors was increased, the variation in motion data from the reference data also increased. Thus it is clear that despite the benefits of filtering, variability still increases as markers are placed further away from the known reference positions.

\section{CONCLUSION}

Variability of kinematic measurements due to inadvertent sensor placement changes was discussed in this paper. It has shown that there is measurement variability due to the failure to place sensors accurately even following same placement protocol for each session. The variability may conceal important motion deviations and meaningful information can be lost. A functional PCA-based technique followed by other techniques was applied on data of the designed experiment. The goal is to compensate for the effects of sensors position changes and having more flexible set-up in terms of sensor placement. Results show differences between similar repetitive actions with random marker position changes and how these variations can be compensated for by applying f-PCA filtering. By keeping the most dominant mode of variation the common motion pattern can be extracted from motion data of several marker wearing sessions. By using the data driven filter, we can realistically derive accurate movement patterns, regardless of random error associated with marker placement.

\section{REFERENCES}

[1] T. Chau, S. Young, and S. Redekop, "Managing variability in the summary and comparison of gait data," J. Neuroeng. Rehabil., vol. 2, pp. 2-22, Jul. 2005.

[2] G. Gorton, D. Hebert, and M. Gannotti, "Assessment of the kinematic variability among 12 motion analysis laboratories," Gait Posture, vol. 29, pp. 398-402, Apr. 2009.

[3] R. Baker, "Gait analysis methods in rehabilitation," J. NeuroEng. Rehabil., vol. 3, pp. 4-5, Mar. 2006.

[4] J. Knight, D. Deen-Williams, T. Arvanitis, C. Baber, S. Sotiriou, S. Anastopoulou, et al., "Assessing the wearability of wearable computers," in Proc. 10th IEEE Int. Symp. Wearable Comput., Oct. 2006, pp. $75-82$.

[5] H. Harms, O. Amft, D. Roggen, and G. Troster, "Rapid prototyping of smart garments for activity- aware applications," J. Ambient Intell. Smart Environ., vol. 1, no. 2, pp. 1-15, 2009.

[6] H. Harms, O. Amft, and G. Troster, "Does loose fitting matter? Predicting sensor performance in smart garments," in Proc. 7th Int. Conf., Sep. 2012, pp. $1-4$.

[7] W. S. Wong and A. Salleo, Flexible Electronics, Materials and Applications. New York, NY, USA: Springer-Verlag, 2009.

[8] R. B. Katragadda and Y. Xu, "A novel intelligent textile technology based on silicon flexible skins," Sens. Actuators A, Phys., vol. 143, pp. 169-174, May 2008.

[9] M. Schartz, J. Trost, and R. Wervey, "Measurment and management of error in quantitative gait data," Gait Posture, vol. 20, no. 2, pp. 196-203, 2004.

[10] U. Croce, A. Leardini, L. Chiari, and A. Cappozzo, "Human movement analysis using streophotogrammetry Part 4: Assessment of anatomical landmark misplacement and its effects on kinematics," Gait Posture, vol. 21, pp. 226-237, Jan. 2005.

[11] U. Groce, A. Cappozzo, and D. Kerrigan, "Pelvis and lower limb anatomical landmark calibration precision and its propagation to bone geometry and joint angles," Med. Biol. Eng. Comput., vol. 37, no. 2, pp. 1029-1034, 1999.

[12] R. Stagni, A. Leardini, A. Cappozzo, M. Benedetti, and A. Cappello, "Effect of hip joint centre mislocation on gait analysis results," $J$. Biomech., vol. 33, no. 11, pp. 1479-1487, 2000.

[13] S. Piazza and P. Cavanagh, "Measurement of the screw-home motion of the knee is sensitive to errors in axis alignment," J. Biomech., vol. 33, no. 8, pp. 1029-1034, 2000.

[14] G. Dona, E. Preatoni, C. Cobelli, R. Rodano, and A. Harrison, "Application of functional principal component analysis in race walking: An emerging methodology," Sport Biomech., vol. 8, no. 4, pp. 284-301, 2010.

[15] L. I. Smith, A Tutorial on Principal Components Analysis. Ithaca, NY, USA: Cornell University, 2002.

[16] A. Donoghue, A. J. Harrison, N. Coeffey, and K. Hayes, "Functional data analysis of running kinematics in chronic achilles tendon injury," Med. Sci. Sports Exercise, vol. 40, no. 7, pp. 1323-1335, 2008.

[17] D. Ormoneit, M. Black, T. Hastie, and H. Kjellstrom, "Representing cyclic human motion using functional analysis," Image Vis. Comput., vol. 23 , no. 14, pp. 1264-1276, 2005.

[18] J. O. Ramsy, G. Hooker, and S. Grave, Functional Data Analysis. New York, NY, USA: Springer-Verlag, 2009.

[19] N. Coffey, A. Harrison, O. Donoghue, and K. Hayes, "Common functional principal components analysis: A new approach to analyzing human movement," Human Movement Sci., vol. 30, pp. 1144-1166, May 2011.

[20] M. Lee, M. Roan, and B. Smith, "An application of principal component analysis for lower body kinematics between loaded and unloaded walking," J. Biomech., vol. 42, no. 14, pp. 2226-2230, 2009.

[21] P. Glardon, R. Boulic, and D. Thalmann, "PCA-based walking engine using motion capture data," in Proc. Comput. Graph. Int., Jun. 2004, pp. 292-298.

[22] A. Daffertshofer, C. Lamoth, O. Meijer, and P. Beek, "PCA in studying coordination and variability: A tutorial," J. Biomech., vol. 19, no. 4, pp. $415-428,2004$.

[23] A. Forner-cordero, O. Levin, Y. Li, and S. P. Swinnen, "Principal component analysis of complex multijoint coordinate movement," Biol. Cybern., vol. 93, no. 1, pp. 63-78, 2005.

[24] C. M. Bishop, Pattern Recognition and Machine Learning. New York, NY, USA: Springer-Verag, 2006.

[25] N. F. Troje, "Decomposing biological motion: A framework for analysis and synthesis of human gait pattern," J. Vis., vol. 2, no. 5, pp. 371-387, 2002. 
[26] K. J. Deluzio and J. L. Astephen, "Biomechanical features of gait waveform data associated with knee osteoarthritis: An application of principal component analysis," Gait Posture, vol. 25, no. 1, pp. 86-93, 2007.

[27] R. Haratian, C. Phillips, and T. Timotijevic, "A PCA-based technique for compensating the effect of sensor position changes in motion data," in Proc. 6th IEEE Int. Conf. IS, Sep. 2012, pp. 126-131.

[28] Codamotion User Guide, Charnwood Dynamics Ltd., Leicestershire, U.K., 2005.

[29] D. Robot, Setting-up Manual, Denso Wave Corp., San Francisco, CA, USA, 2011.

[30] J. Hamill, and W. Selby, Three Dimensional Kinematics in Research Methods in Biomechanics, D. G. E. Robertson, G. E. Caldwell, J. Hamill, G. Kamen, and S. N. Whittlesey, Eds. Champaign, IL, USA: Human Kinetics, 2004.

[31] L. Trefethen and D. Bau, Numerical Linear Algebra. Philadephia, PA, USA: Soc. Ind. Appl. Math., 1997.

[32] V. Zatsiorsky, Kinematics of Human Motion, Champaign, IL, USA: Human Kinetics, 1998.

[33] N. Boulgouris, K. Plataniotis, and D. Hazinakos, "Gait recognition using linear time normalization," Pattern Recognit., vol. 39, no. 5, pp. 969-979, 2006.

Roya Haratian (SM'11) is currently working towards the Ph.D. degree at the School of Electronic Engineering and Computer Science, Queen Mary University of London, London, U.K. Her research interest is sensor networks, resource allocation, signal processing and machine learning. Prior to her Ph.D. degree, she pursued the B.Sc. and M.Sc. degree in electronic engineering and communications systems, respectively, from the Isfahan University of Technology and Tarbiat Modares University, Tehran, Iran. Before joining the University of London as a full time researcher, she was working as a Researcher at the Iran Telecommunication Research Centre and lecturer in technological Institutes. She is currently Chair of the IEEE Women in Engineering Group, Queen Mary University of London.

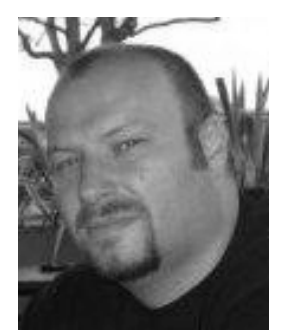

Richard Twycross-Lewis was born in LeamingtonSpa, Great Britain, in 1976. He received the B.Sc. (Hons) degree in sport and exercise science from London South Bank University in 1999, the M.Res. degree in advanced instrumentation systems from University College London in 2000, and the Ph.D. degree in medical engineering from Queen Mary University of London in 2005. In 2008, he joined the Centre for Sports and Exercise Medicine (CSEM), Queen Mary University of London, as a Research Supervisor. Since January 2010, he has been lecturer and module leader for the undergraduate and postgraduate biomechanics modules within CSEM. His current research interests include gait analysis in children with cerebral palsy, biomechanics in Olympic lifting and wavelet analysis in multichannel surface electromyography. Dr. Twycross-Lewis is a Member of the Institute of Physics, the Institute of Physics and Engineering in Medicine, the British Association of Sport and Exercise Sciences, and The National Strength and Conditioning Association.
Tijana Timotijevic received the B.Eng. degree in electronic and electrical engineering from Brunel University, London, U.K., in 1994, the Ph.D. degree in telecommunications from Queen Mary, University of London, U.K., in 1999, and the M.Sc. degree in biomedical engineering from the University of Surrey, Guildford, U.K., in 2007. From 1998 to 2001, she was a System Engineer with Bell Labs and Avaya Communications R\&D, U.K. In 2001, she joined Technology Development of Vodafone U.K. as a Principal Engineer, where she worked on design and development of Vodafone wide area packet network. Between 2003-2005, she managed WAN design and service integration and planning teams in Vodafone U.K. In 2005, she worked at Vodafone Global Services (Networks) as a Senior Manager. Since 2008, she has been with Queen Mary, University of London, U.K., where she is now a Lecturer of Electromagnetics and Bioelectromagnetics. Her research interests include motion capture for assisted rehabilitation, use of travelling wave dielectrophoresis for steering neuronal growth, and study of mechanisms of neuronal interactions with electromagnetic fields.

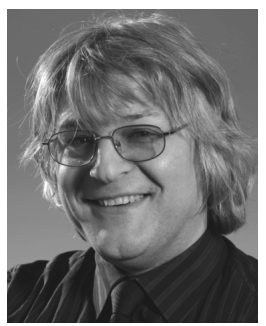

Chris Phillips received the B.Eng. degree in telecommunications engineering from Queen Mary, University of London, in 1987, followed by the $\mathrm{Ph} . \mathrm{D}$. degree in concurrent discrete event-driven simulation, also from Queen Mary. He then worked in industry for nine years as a Hardware and Systems Engineer with Bell Northern Research, Siemens Roke Manor Research and Nortel Networks, focusing on broadband network protocols, resource management and resilience. In 2000, he Chris returned to Queen Mary. A key theme that underpins his recent research is how mechanisms can be developed to enable resources to be used effectively in a changing or uncertain environment, particularly in regard to sensor networks. 